\title{
Dispelling superstitions in Nepalese society with astronomy
}

\author{
Rishi Shah \\ Nepal Academy of Science and Technology (NAST), \\ c/o H.B. Complex, Lalitpur-4, GPO Box 3459, Kathmandu, Nepal \\ email: rishishah@enet.com.np
}

\begin{abstract}
Throughout human history, astronomy has played crucial rôle in the development of our civilization, culture and daily chores of lives that have been influenced by observations of Sun, moon, planets, stars and other cosmic entities. Our ancestors who were hunting and gathering and foraging food while living in caves learned to think logically by gazing at the twinkling stars in the heavens. Seasons for crops plantation were determined, time concept was introduced, entire sky was charted and the motions of celestial objects were meaningfully understood. With the advent of telescopes, the geocentric model of universe was replaced by the revolutionary heliocentric concept of our Solar System. Astronomy dispelled superstitious beliefs strongly prevailing in societies. Closely associated with numerous disciplines of science astronomy is still flourishing worldwide and is attempting to fly us away to those habitable cosmic bodies of our universe. By establishing well-equipped observational infrastructure local and international astronomy research and development could be enhanced. Introduction of astronomy in education system right from school would attract and encourage students to pursue higher studies for enabling them for participating in future international scientific and exploration programmes. Astronomy has helped our society to progress peacefully and efficiently.
\end{abstract}

Keywords. Society, astrology, supersitition, Nepal

\section{The situation of astronomy in Nepalese society}

Nepal is a mountainous country that is blessed with uniquely plentiful natural beauties enriched with enchantingly diverse flora, fauna and culture. It is the home of world's highest Himalayan Mountains including Mount Everest with rough and rugged terrain. Circa eighty percent of Nepalese population is fully dependent on agriculture that dwells significantly (fairly $72 \%$ ) in the rural areas. Agriculture is the backbone of country's economy. Its GDP is 12.7 Billion USD in 2008. As a developing country its average per capita income indicates merely 460 USD. Its population is about 29 million. Education is free till tenth grade in government schools.

In Nepal astrological rituals are practiced vehemently throughout the life of a person. From birth till death most of daily lives is influenced by beliefs that have origin in astrology, as the society members fully believe that one's fate or destiny is predetermined by some superior celestial power inhabiting the sky. Life's course is already fixed and one has to follow every day routine without any questions, as guided by the instinct just to pass through the time of life, where slight maneuver from predestined path is possible by appeasing some planets. These types of deep-rooted beliefs have been handed down from one to next generations and have been accepted as justification for good or dismal performance in one's life. Many ethnical groups share similar appallingly inherent thoughts. Almost every Hindu possesses a horoscope that describes the person's life cycle. They are written cleverly by astrologers and priests who generally command strong social status and position in the society. 
Nepal is geographically squeezed between two big nations India and China that have currently aspired over-all devolvement even though they have inherited traditional and classical ways of pondering and mulling particularly on human existence and on philosophy of life. One could argue that their success was spurred by the inclusion of modem education that embolden the younger generation to contemplate and be innovative for moving with time to adopt themselves to the new challenges rather than believing blindly on their destiny.

Thus, if a nation would formulate an education system that could adorn young students with objective ways of analysis for tackling problems in any sector, its progress would be undoubtedly guaranteed. One of the subjects that could really contribute to this process of new postulation would be astronomy, because astronomy is related to multi-faceted subjects as mathematics, science and sociology. Innovative change is the most stable factor for our existence. Nurturing talents of prodigies require optimum investments on institutions with capable personals that have specialized to train and impart confidence to naturally gifted persons for accessing and utilising their ingenuity for meaningful purposes for the benefit of the community. Formal education with astronomy at schools could be the starting point for future innovators to be trained for releasing their hidden creative strength.

Programmes on astronomy that have been executed currently by some reputed schools in Nepal have exposed younger generation to become more constructive and innovative, while attempting to address vexing and worrisome issues. Although basic astronomy for schools is evidently present but the instruction methodologies need to be seriously embellished and upgraded with time. It is imperative that extra-curricular activities on astronomy like viewing of heavenly bodies would emulate the nature and standard astronomy education considerably. Astronomy could be even suggested at various levels of education throughout Nepal in future.

Astronomy is one of the oldest sciences taught and practiced by Nepali culture. Our astronomers had watched the heavenly wonders relatively well in detail. They interpreted the findings quite accurately. Astronomical artifacts and writings bear testimony to their actions. Astronomy (both theoretical and observational) served as corner stone for planning crop harvest, movement of goods, making of lunisolar calendars, ephemeris and horoscopes (that are based mostly on astrology) and conduction of countless religious rituals that govern one's day-to-day behavior. The conventional astronomy and its interpretations still form the dominating base for our ways of lives. The modern disclosures have not fully been adopted in the current classical teachings, although efforts are emphatically underway even with limited financial and human resources.

Any extra-curricular astronomical initiations could appreciate the astronomy education at basic and higher levels, as the acceptance and interest in the students, family and society is ever increasing. Amateur astronomers like the members of our group have handled awareness programmes on astronomy that are implemented regularly with great participation of schools' faculty members, public and government officials. Discerning and recognising the Moon, stars, comets, meteor showers and deep-sky objects such as star clusters, galaxies, nebulae, pulsars and supernovae have become very popular. Astrophotography has reinforced team work exercises.

Although astronomy has made tremendous strides in knowing the nature of the universe and its contents, answers to origin of universe, dark matter and dark energy probably influencing evolution and fate of the inflating cosmos (Big Bang and Hubble's Law), cosmic microwave background radiation, Fermi paradox, evolution of our lives and our eventual fate on earth are always contentious subjects for discussions. These exercises 
have facilitated philosophical polemics as required for deeper comprehending our existence and for negating superstitious beliefs that we have been exposed to.

\section{Astronomy for dispelling superstitious beliefs}

Leading organizations as NASA, ESA, ISRO, JAXA, RSA, CNSA etc are running distinguished space explorations where international collaboration could be endorsed and underscored. With proper investment in infrastructure (like modern observatory with other ancillary facilities) could provide backbone for mutual astronomy programmes as satellite tracking, observations of planets and deep sky objects along with monitoring space weather and transient events with astro photography that could be economically lucrative and interesting. Employment for younger professional could be offered. Rural development would be triggered during setting-up of such establishments.

Institutions for formal astronomy education with curriculum and matching pedagogic tools at university, campus and school are being introduced in Nepal. BP Koirala Memorial planetarium and Observatory Project (see http://www. planeta-observatory.gov . $\mathrm{np}$ ) belonging to Ministry of Science and Technology is generously active for astronomical pursuits. As many public-private institutes and NGOs have been handling many astronomy endeavours professionally, they are being empowered and mobilised emphatically.

Since the need for stressing the importance of astronomy in our country is indispensable as superstitious beliefs are glaringly present in our society and culture, rational elucidations of celestial phenomena would surely reduce the blind faith and beliefs that have been affecting (mostly negatively) our lives. NASO's awareness programmes on stars, planets and other entities of universe with proper elucidations of bewildering phenomena as eclipses and mysteries are being managed at appropriate time intervals. They have contributed extensively for augmenting and uplifting rational judgement process of Nepalese people and especially of younger generation. Special tailored-made projects on astronomy for teachers and journalists are being energetically organised by the Nepal Astronomical Society (NASO, see http://astronomy-nepal.blogspot.com).

TheNepal Academy of Science and Technology (NAST, see http://www.nast.org.np) has been promoting such astronomy activities from the time when astronomy projects began enthusiastically in 1986 with the observation of Halley's Comet. Interactions with press and public and with students are still being carried out. NAST's Science Popularisation Project (SPP) with donor and local support has ushered in quest for bringing S\&T nearer to the people at large via TV, radio and print media. Its outset programme had commenced with Space Shuttle's lift-off and is still going on dynamically till today. Logical and reasonable ways of thinking for solving problems that hinder our social, economical and technical progress could be s ubstantially achieved by beginning astronomy in academic institutions as schools and by bringing astronomy to people at large for dispelling superstitions beliefs in our society in order to obtain peace and prosperity for improving our quality of our lives. 